\title{
Pushing the limits of crystallography with EIGER
}

\author{
M. Müller and A. Förster \\ DECTRIS Ltd., Täfernhof 1, 5405 Baden-Dättwil, Switzerland \\ marcus.mueller@dectris.com
}

With the recently introduced EIGER, hybrid photon counting (HPC) enters a new dimension of spatial and temporal resolution and expands the field of x-ray experimentation. A pixel size of $75 \mu \mathrm{m}$ and continuous read-out with auto-summation increases the quality of the collected data. Frame rates of up to $750 \mathrm{~Hz}$ enable new ways of doing $\mathrm{x}$-ray crystallography like accurate positioning of microcrystals by diffraction and synchrotron serial crystallography. EIGER R $1 \mathrm{M}$ and $4 \mathrm{M}$ are mega-pixel HPC detectors for the laboratory that are based on the same technology as the most powerful detectors available at select synchrotron beamlines. The absence of any detector noise in combination with an image bit depth of 32 bit and high spatial resolution turn them into versatile platforms for hitherto unthinkable laboratory application.

A short outline of the differences between EIGER and PILATUS3 will highlight key aspects of the new detector technology and show that higher data quality can be achieved with EIGER [1]. Examples from several beamlines will illustrate ways of setting up serial crystallography $[2,3]$ and grid scanning approaches [4], especially with an eye on increasing signal to noise from microcrystals. Data obtained from two protein arginine methyltransferases and a prokaryotic ribosome demonstrate the power of the home source where, in addition, the long wavelength ensures that significant native anomalous signal is almost always measured, with benefits for experimental phasing, molecular replacement and metal ion identification.

1. A. Casanas et al., Acta Cryst. D72, (2016), 1036-48

2. P. Roedig et al., Sci Rep. 5 (2015) 10451

3. N. Coquelle et al., Acta Cryst. D71, (2015), 1184-96

4. U. Zander et al., Acta Cryst. D71, (2015), 2328-43. 